\title{
WAVELET TRANSFORM AND LIP MODEL
}

\author{
GuY COURBEBAISSE ${ }^{1}$, FREDERIC TRUNDE ${ }^{2}$ AND MICHEL JOURLIN ${ }^{2}$
}

${ }^{1}$ Ecole Supérieure de Plasturgie - 85, rue Henri Becquerel 01100 Bellignat, ${ }^{2}$ Ecole Supéieure de Chimie Physique Electronique de Lyon - Laboratoire LISA - 43, bd du 11 novembre 1918 - 69616 Villeurbanne e-mail: courbebaisse@cpe.fr

(Accepted May 21, 2002)

\begin{abstract}
The Fourier transform is well suited to the study of stationary functions. Yet, it is superseded by the Wavelet transform for the powerful characterizations of function features such as singularities. On the other hand, the LIP (Logarithmic Image Processing) model is a mathematical framework developed by Jourlin and Pinoli, dedicated to the representation and processing of gray tones images called hereafter logarithmic images. This mathematically well defined model, comprising a Fourier Transform "of its own", provides an effective tool for the representation of images obtained by transmitted light, such as microscope images. This paper presents a Wavelet transform within the LIP framework, with preservation of the classical Wavelet Transform properties. We show that the fast computation algorithm due to Mallat can be easily used. An application is given for the detection of crests.
\end{abstract}

Keywords: logarithmic image processing, logarithmic wavelet transform, wavelet transform.

\section{INTRODUCTION}

Jourlin and Pinoli (1984) introduced a new mathematical approach called Logarithmic Image Processing (LIP), providing a framework for representation and processing of gray tone functions i.e., logarithmic images with gray levels in a bounded range.

In the first published paper on the LIP model (Jourlin and Pinoli, 1988), a new algebraic structure has been presented for image processing. The authors represent an image by a light filter through which the light passes to form an image. The absorption of the light filter is called the gray tone function. Moreover, Jourlin and Pinoli (1996) show that the LIP model is consistent with the non linear logarithmic human visual system. The link between human visual images and transmitted light images appears accordingly in a natural way (Jourlin and Pinoli, 1996).

Pinoli (1992) proposed then a consistent mathematical theory wherein the main operations are: addition $\Delta$, subtraction $\Delta$, multiplication by a scalar $\Delta$, multiplication of gray tone functions $A$ differentiation, complemented by two operations of great interest for this paper: integration and convolution. The LIP model gives to a set of transmitted light images the status of a vector space structure, denoted $\mathbf{G}$, with an additive law $\triangle$ and a multiplicative law $\Delta$ (Jourlin and Pinoli, 1988; 1996):

$$
\begin{aligned}
& \mathbf{f} \Delta \mathbf{g}=\mathrm{f}+\mathrm{g}-\left(\frac{\mathrm{fg}}{\mathrm{M}}\right) \text { and } \\
& \beta \Delta \mathbf{f}=\mathrm{M}-\mathrm{M}\left(1-\frac{\mathrm{f}}{\mathrm{M}}\right)^{\beta},
\end{aligned}
$$

where $\mathbf{f}$ and $\mathbf{g}$ (bold type) functions are gray tone functions defined on the bounded spatial support [0,M[ (0: no absorption, M: totally opaque image). The functions $f$ and $g$ are usual gray tone functions (i.e. when the logarithmic function is used with ordinary operations such as + ). $\alpha$ is a positive scalar. $\mathrm{M}$ is the maximum gray tone.

There is a one to one relation, noted $\zeta$, between space $\mathbf{G}$ and the space of the usual functions defined on the spatial support D (Pinoli, 1992). $\zeta$ is expressed as:

$$
\begin{aligned}
& \Psi: \mathbf{f} \rightarrow \Psi(\mathbf{f})=-\mathrm{M} \ln \left(1-\frac{\mathrm{f}}{\mathrm{M}}\right) \text { and } \\
& \Psi^{-1}: \mathrm{h} \rightarrow \Psi^{-1}(\mathrm{~h})=\mathrm{M}\left(1-\exp \left(\frac{-\mathrm{h}}{\mathrm{M}}\right)\right)
\end{aligned}
$$

$\Psi$ is an isomorphism of vector structures between space $\mathbf{G}$ and the usual functions with complex values in the spatial support D. This isomorphic transform $\Psi$ is a powerful tool for developing the LIP model. As a matter of fact, the isomorphism $\Psi$ allows to build 
operations on gray tones such as dot product between gray tone images, LIP model integration, LIP model Fourier transform (Jourlin and Pinoli, 1996). This powerful approach has been applied with a large success in different fields.

\section{LOGARITHMIC WAVELET TRANSFORM IN THE G SPACE}

Multi-resolution analysis, till now, has not been explored with the aid of the LIP model. In this paper, we show how the Wavelet transform can be defined in the LIP space. Then, we give some numerical applications. After a brief recall about the classical Wavelet Transform (WT), the Logarithmic Wavelet Transform (LWT) is addressed with special care given to the admissibility properties (Torrésani, 1995).

\section{Classical wavelet and wavelet transform definitions}

Consider the function $\wp(\mathrm{x})$, called mother wavelet, where $\mathrm{x} \in \mathbb{C}^{2} ; \mathrm{b} \in \mathbb{C}$ and $\mathrm{a} \in \mathbb{C}_{+}^{*}$ :

$$
\wp_{(a, b)}(x)=\frac{1}{a^{2}} \wp\left(\frac{x-b}{a}\right),
$$

$\wp_{(a, b)}$ is dilated by a scale 'a', and translated by a 'b' delay.

One defines the Wavelet Transform of a function $f$ belonging to $L^{2}(\mathbb{C})$ by:

$$
\mathrm{W}(\mathrm{a}, \mathrm{b})(\mathrm{f})=\frac{1}{\mathrm{a}^{2}} \int_{\mathrm{C}^{2}} \mathrm{f}(\mathrm{x}) \wp^{*}\left(\frac{\mathrm{x}-\mathrm{b}}{\mathrm{a}}\right) \mathrm{dx},
$$

where $\wp^{*}$ is the conjugate expression of $\wp$,

i.e.,

$$
\mathrm{W}(\mathrm{a}, \mathrm{b})(\mathrm{f})=\left(\mathrm{f}(\mathrm{x}) \mid \wp_{(\mathrm{a}, \mathrm{b})}(\mathrm{x})\right)_{\mathrm{L}^{2}(\mathrm{C})},
$$

where a is a scale factor, $\mathrm{b}$ is a position parameter and $\wp_{(\mathrm{a}, \mathrm{b})}$ is the analyzing wavelet (Torrésani, 1995; Mallat, 1999).

\section{Logarithmic wavelet definition}

In the LIP context, the logarithmic wavelet $\wp_{\Delta}$ associated to $\wp$, is defined by the function $\wp_{\Delta}=\Psi^{-1}(\wp)$ in the complex case. In a developed form, it comes:

$$
\wp_{\mathrm{E}(\mathrm{a}, \mathrm{b})}(\mathrm{x})=\frac{1}{\mathrm{a}^{2}} \Delta \Psi^{-1}\left(\wp\left(\frac{\mathrm{x}-\mathrm{b}}{\mathrm{a}}\right)\right) .
$$

Admissibility conditions impose a zero average to the wavelet. One can show that the LIP wavelet associated to a zero average wavelet has itself a zero average:

$$
\begin{aligned}
& \int_{D} \wp(x) d x=0 \Rightarrow \Psi^{-1}\left(\int_{D} \wp(x) d x\right)=0 \Rightarrow \\
& \int_{D} \Psi^{-1}(\wp)(x) d x=0 \Rightarrow \int_{D} \wp_{\Delta}(x) d x=0 .
\end{aligned}
$$

\section{Logarithmic wavelet transform definition}

As an extension of the classical Wavelet Transform, the Logarithmic Wavelet Transform of a function $\mathbf{f}$ is defined by:

$$
\mathbf{W}_{\Delta}(\mathrm{a}, \mathrm{b})(\mathbf{f})=\int_{\mathrm{C}^{2}} \wp_{\Delta}{ }^{*}(\mathrm{a}, \mathrm{b})(\mathrm{x}) \otimes \mathbf{f}(\mathrm{x}) \mathrm{dx} .
$$

The isomorphism $\Psi$ preserves the wavelet transform, i.e.:

$\forall \mathbf{f} \in \mathrm{L}^{2}, \mathrm{~W}(\mathrm{a}, \mathrm{b})(\Psi(\mathbf{f}))=\Psi\left(\mathbf{W}_{\Delta}\right)(\mathrm{a}, \mathrm{b})(\mathbf{f})$,

where $\mathrm{W}(\mathrm{a}, \mathrm{b})$ is the classical wavelet transform. When $\mathbf{f}$ is a logarithmic image :

$$
\mathbf{W}_{\Delta}(\mathrm{a}, \mathrm{b})(\mathbf{f})=\Psi^{-1}(\mathrm{~W})(\mathrm{a}, \mathrm{b})(\Psi(\mathbf{f})) .
$$

This demonstrates that the Logarithmic Wavelet Transform of $\mathbf{f}$ can be calculated directly from the classical Wavelet Transform of $\Psi(\mathbf{f})$ (Trunde et al., 2001).

\section{Discrete wavelet transform applied to image analysis}

In our application, a Mallat's horizontal and vertical analysis is performed (Mallat, 1999). This two-dimensional algorithm is based on the separation of variables leading to a prioritization of the horizontal and vertical directions. The wavelet transform can be interpreted as a frequency decomposition where each set has a particular orientation. Fig. 1 illustrates the different steps of the corresponding analysis (decomposition) and of the synthesis (reconstruction). 


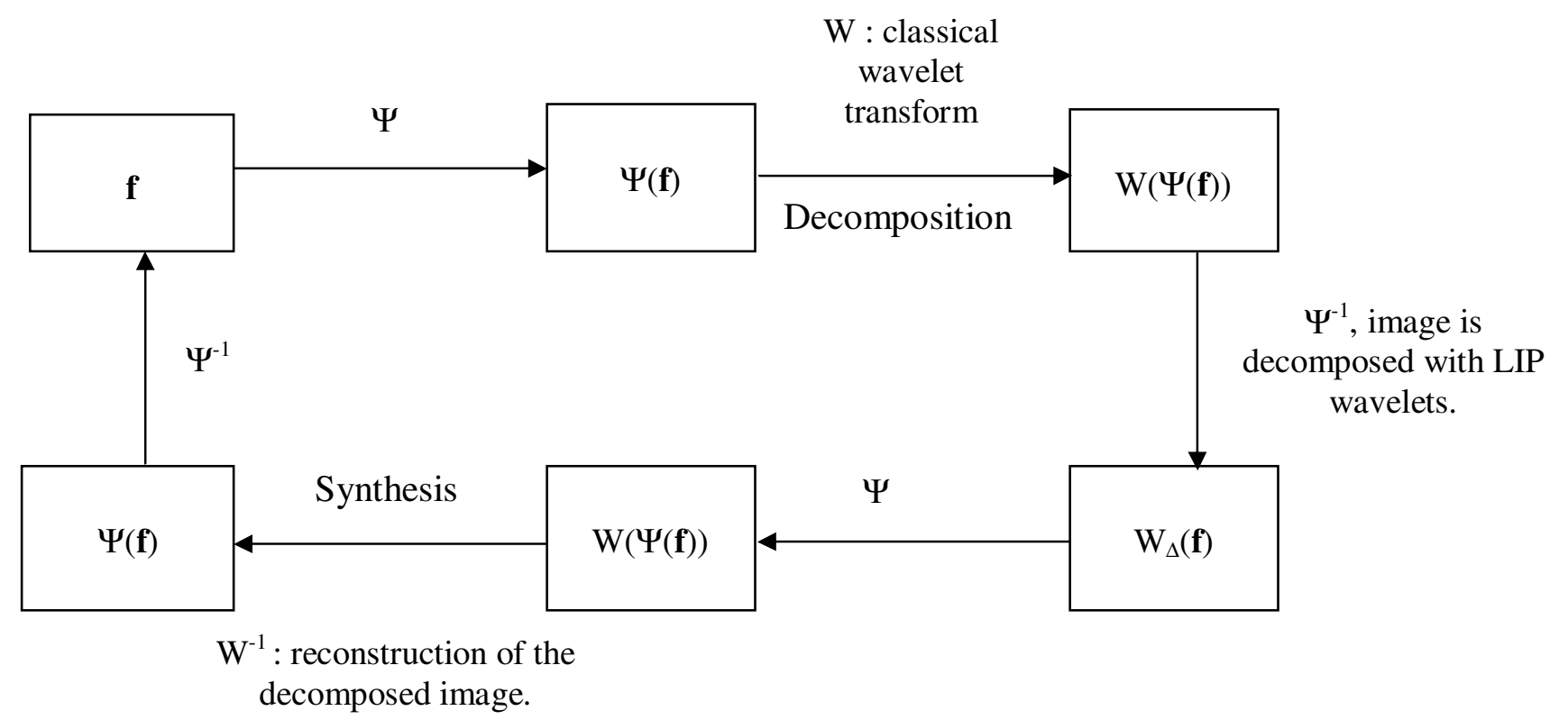

Fig. 1. Steps of the Logarithmic Wavelet Transform.

\section{APPLICATION}

An example is considered below to illustrate the Logarithmic Wavelet Transform computation. One uses here the Daubechies's wavelet which forms an orthonormal basis (Mallat, 1999). The original image (Fig. 2) is an aerial view where we want to pick up the main crest.

In order to highlight the specific effects of the Logarithmic Wavelet Transform, in comparison to results obtained with the Classical Wavelet Transform, our example is first treated in one dimension. Then, the analysis is extended to the two-dimensional case in order to confirm the effects illustrated in the 1D case.

\section{D CASE : EXAMPLE OF DECOMPOSITION}

The 1D Wavelet Transform of the selected cut is computed with a Daubechies'mother wavelet (Daubechies, 1988). The decomposition is performed through 4 scales [a: $a_{0}$ to $a_{3}$ ]. The wavelet coefficients (Fig. 3) i.e., the output of the wavelet transform computation at each scale, can be interpreted in term of a frequency analysis where $\mathrm{a}_{0}$ corresponds to a low frequency component and $\mathrm{a}_{3}$ to a high frequency component of the considered signal. Fig. 3 shows the decomposition by a classical WT (Fig. 3, left) and by the LWT (Fig. 3, right).
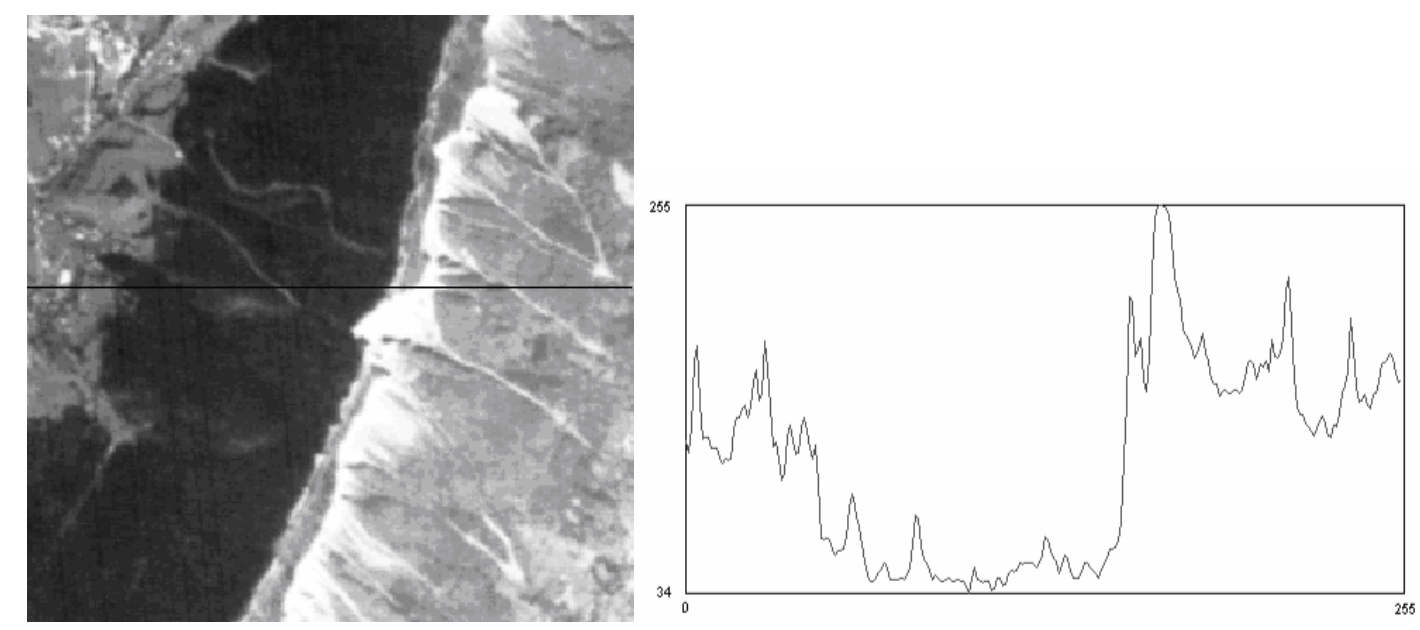

Fig. 2. Original image (left) and ID signal (signal) corresponding to the cut on the left image (magnitude (vertical axis) versus dimensionless unit of length (horizontal axis). 
The LIP decomposition on four scales (Fig. 3) of the signal in Fig. 2 (right), gives an almost optimal visualization of the dynamic range of the wavelets coefficients. Some of the characteristics of the signal are accordingly underlined more efficiently.

In order to appreciate which of the signal elements are stressed by the LWT, some frequencies are eliminated. Then, the characteristics of the reconstructed signal are examined.

The signal (Fig. 2, right) is now decomposed on

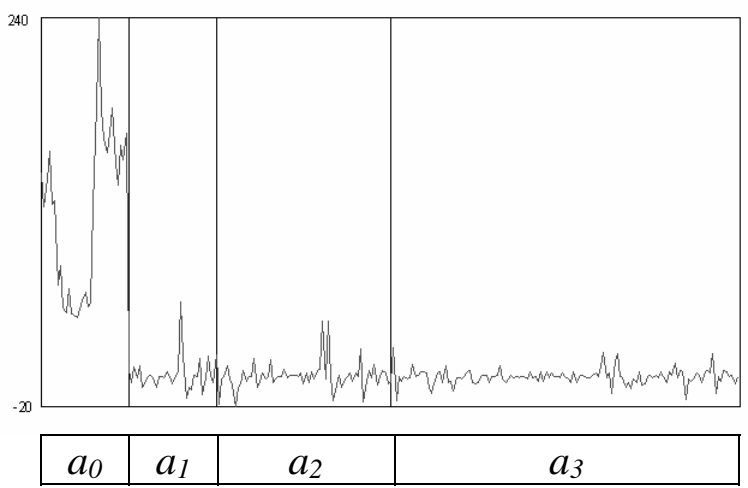

six scales $\left(a_{0}\right.$ to $\left.a_{5}\right)$. Fig. 4 (left) shows the WT case and Fig. 5 (left) shows the LWT case.

The low frequency coefficients of the decomposition are then set to zero. After synthesis (Inverse Wavelet Transform), the high frequency components are found (Figs. 4 and 5, right). The net result is that particular elements in the signal are strongly enhanced (Fig. 5, right). In our example, these particular elements correspond to strong signal transitions i.e., crest in the higher range of the signal (values near M).

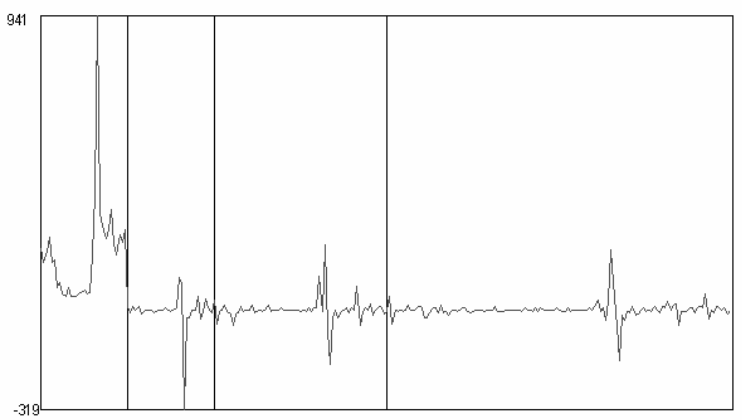

Fig. 3. ID case: Classical decomposition by WT and LIP decomposition by LWT (Wavelet coefficient (vertical axis) magnitude versus scales (horizontal axis)

WT case:
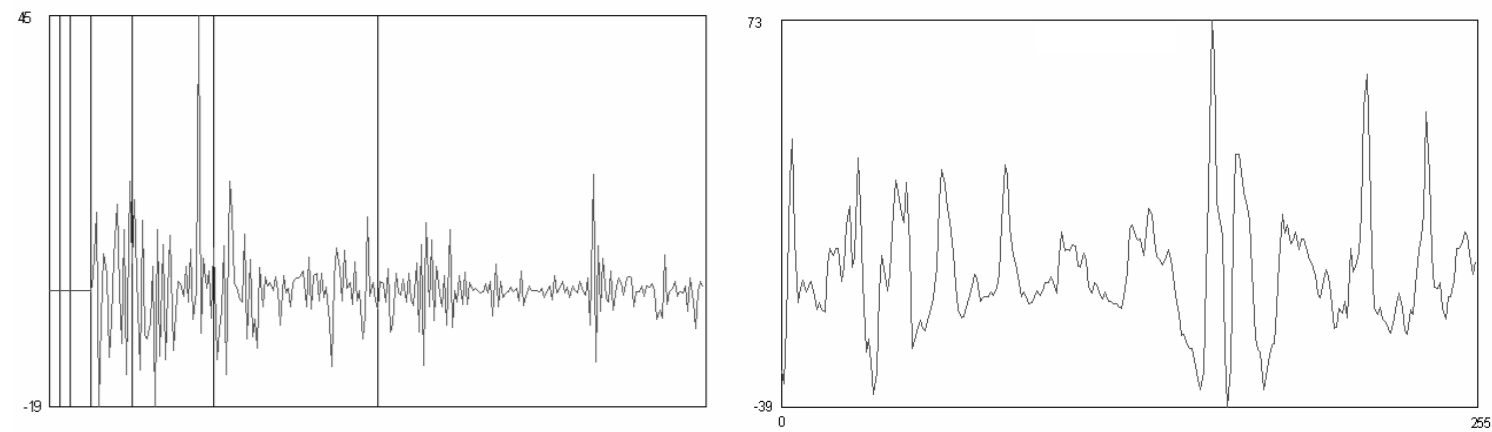

Fig. 4. WT coefficients of the signal with low frequency components inhibition (left) and Reconstruction: Magnitude versus dimensionless unit of length (right).

LWT case :
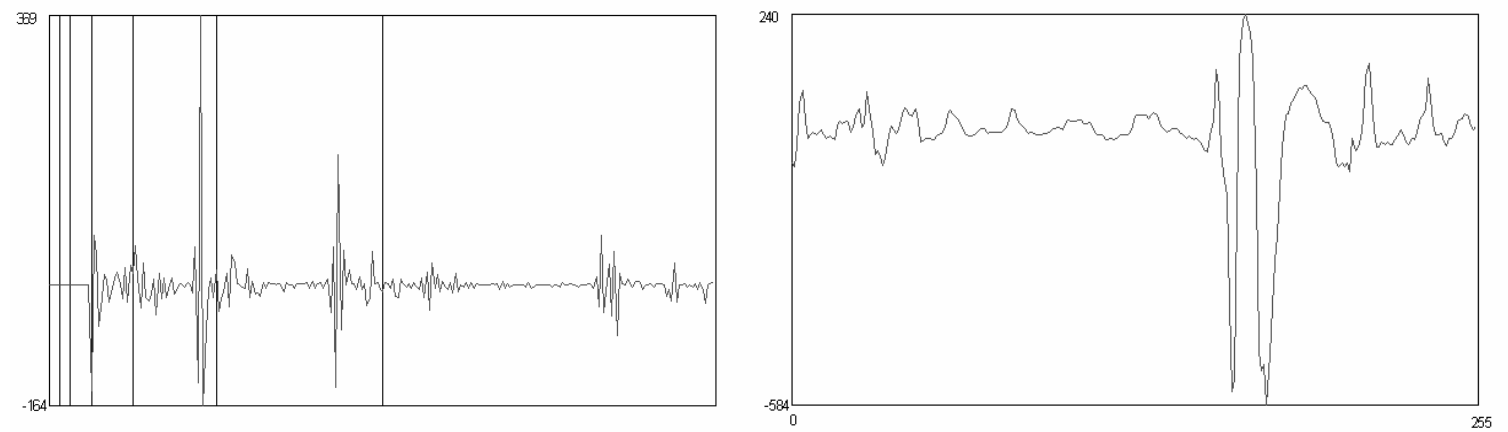

Fig. 5. LWT coefficients of the signal with low frequency components inhibition (left) - Reconstruction: Magnitude versus dimensionless unit (right). 

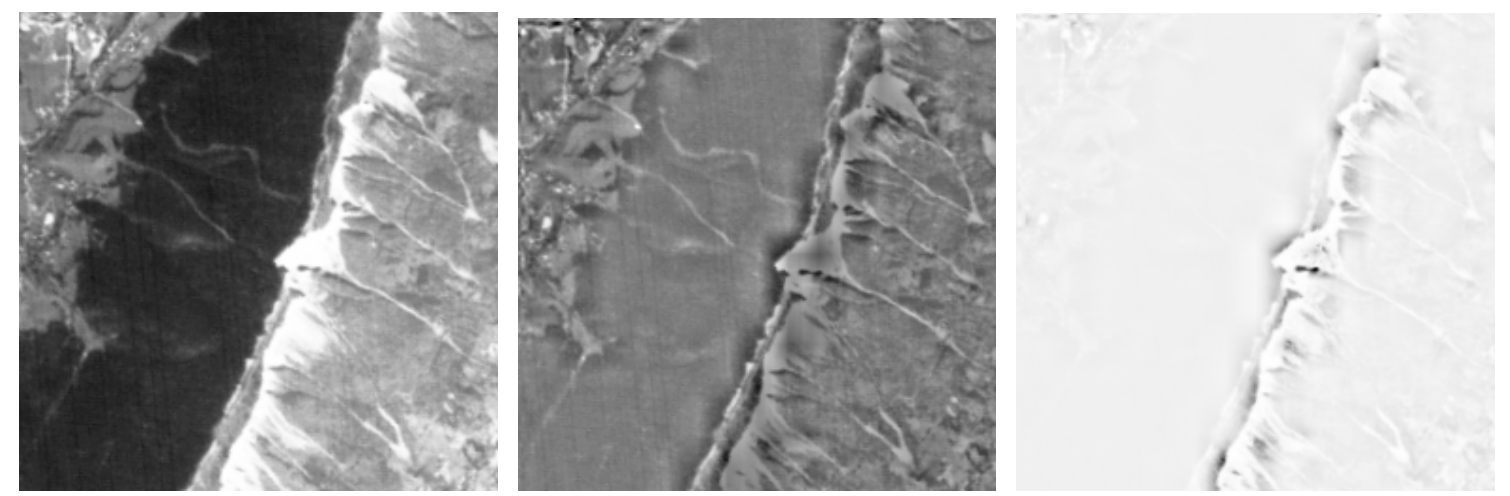

Fig. 6. Original image (left), WT (middle) versus LWT (right).

\section{D CASE}

We now consider the whole image (Fig. 2 or 6 , left). The 2D wavelet transform is computed (the parameters constraining the decomposition and the synthesis are identical to the 1D case).

Fig. 6 (middle) gives the final result in the classical WT configuration and Fig. 6 (right) the final result in the LWT configuration. One can see on the Fig. 6 (right) that the mountain crest of the original image has been enhanced as opposed to the rest of the image where the gray levels have been leveled.

\section{CONCLUSION}

This paper has introduced the Logarithmic Wavelet Transform and shows that it can be computed by means of the classical Wavelet Transform and the isomorphic transform $\Psi(\mathbf{f})$. Our application gives a first idea of the type of process that can be performed in order to point out particular details in an image such as lines of crest. The LIP model associated to the Wavelet Transform emphasizes certain features such as transitions in a specific range of gray level that are not enhanced using classical Wavelet Transform. The LWT concept brings new perspectives, and represents a whole field for future developments in image processing.

\section{REFERENCES}

Daubechies I (1988). Orthonormal Bases of Compactly Supported Wavelets. Pure Appl Math 4:909-96.

Jourlin M, Pinoli JC (1984). Morphologie des fonctions de gris: une nouvelle approche. Technical report. Signal processing laboratory and department of mathematics, Universitéde St-Etienne, 21 pages.

Jourlin M, Pinoli JC (1988). A Model for Logarithmic Image Processing. Journal of Microscopy 149(1):21-35.

Jourlin M, Pinoli JC (1996). Justifications physiques et applications du modde LIP pour le traitement des images obtenues en lumiòre transmise. Traitement du Signal 3:251-62.

Mallat S (1999). A Wavelet Tour of Signal Processing. New York: Academic Press.

Pinoli JC (1990). Le modde LIP VII: transformation de Fourier. Rapport 7. Département de mathématiques, Universitéde St-Etienne, 15 pages.

Pinoli JC (1992). Modaisation et traitement des images logarithmiques: théorie et applications fondamentales. Département de mathématiques, Université de StEtienne.

Torrésani B (1995). Analyse continue par ondelettes. Inter Editions, CNRS Editions.

Trunde F, Courbebaisse G, Jourlin M (2001). Logarithmic Wavelet Transform. Proc. $8^{\text {th }}$ ECS and Image Analysis, (Bordeaux), 293-8. 
